\title{
Enhancement in Tonically Active Glutamatergic Inputs to the Rostral Ventrolateral Medulla Contributes to Neuropathic Pain-Induced High Blood Pressure
}

\author{
Wei Wang, ${ }^{1}$ Zui Zou, ${ }^{2}$ Xing Tan, ${ }^{3}$ Ru-Wen Zhang, ${ }^{3}$ Chang-Zhen Ren, ${ }^{3}$ Xue-Ya Yao, ${ }^{4}$ \\ Cheng-Bao Li, ${ }^{4}$ Wei-Zhong Wang, ${ }^{3}$ and Xue-Yin Shi ${ }^{1}$ \\ ${ }^{1}$ Department of Anesthesiology and SICU, XinHua Hospital, Shanghai JiaoTong University School of Medicine, \\ Shanghai 200092, China \\ ${ }^{2}$ Department of Anesthesiology, Changzheng Hospital, Second Military Medical University, Shanghai 200433, China \\ ${ }^{3}$ Department of Physiology, Second Military Medical University, Shanghai 200433, China \\ ${ }^{4}$ Hebei North University, Zhangjiakou, Hebei Province 075000, China
}

Correspondence should be addressed to Wei-Zhong Wang; wangwz68@hotmail.com and Xue-Yin Shi; shixueyin1128@163.com

Received 1 April 2017; Revised 19 June 2017; Accepted 10 July 2017; Published 12 October 2017

Academic Editor: Depei Li

Copyright (c) 2017 Wei Wang et al. This is an open access article distributed under the Creative Commons Attribution License, which permits unrestricted use, distribution, and reproduction in any medium, provided the original work is properly cited.

\begin{abstract}
Neuropathic pain increases the risk of cardiovascular diseases including hypertension with the characteristic of sympathetic overactivity. The enhanced tonically active glutamatergic input to the rostral ventrolateral medulla (RVLM) contributes to sympathetic overactivity and blood pressure (BP) in cardiovascular diseases. We hypothesize that neuropathic pain enhances tonically active glutamatergic inputs to the RVLM, which contributes to high level of BP and sympathetic outflow. Animal model with the trigeminal neuropathic pain was induced by the infraorbital nerve-chronic constriction injury (ION-CCI). A significant increase in BP and renal sympathetic nerve activity (RSNA) was found in rats with ION-CCI (BP, $n=5$, RSNA, $n=7, p<0.05)$. The concentration of glutamate in the RVLM was significantly increased in the ION-CCI group ( $n=4$, $p<0.05)$. Blockade of glutamate receptors by injection of kynurenic acid into the RVLM significantly decreased BP and RSNA in the ION-CCI group $(n=5, p<0.05)$. In two major sources (the paraventricular nucleus and periaqueductal gray) for glutamatergic inputs to the RVLM, the ION-CCI group $(n=5, p<0.05)$ showed an increase in glutamate content and expression of glutaminase 2, vesicular glutamate transporter 2 proteins, and c-fos. Our results suggest that enhancement in tonically active glutamatergic inputs to the RVLM contributes to neuropathic pain-induced high blood pressure.
\end{abstract}

\section{Introduction}

Neuropathic pain is widely recognized as one of the most difficult pain syndromes to manage, and its outcomes are often unsatisfactory. Neuropathic pain is a risk factor for cardiovascular diseases, such as hypertension, diabetes, and stroke, seriously affects the quality of patients' life, and results in the higher anxiety/depression scores [1-6]. Neuropathic pain is capable of increasing blood pressure (BP) and heart rate (HR) $[3,4,7]$. However, the mechanism by which neuropathic pain induces cardiovascular dysfunction is not fully understood.
A major characteristic of cardiovascular diseases including hypertension and heart failure is overactivity of the sympathetic nervous system (SNS). Increasing clinical evidence indicates a key role for sympathoactivation in the development of these cardiovascular diseases $[8,9]$. Interestingly, neuropathic pain is suggested to be a stressor for stimulating the SNS [10]. The rostral ventrolateral medulla (RVLM) is a key region involved in the central control of sympathetic outflow and plays an important role in maintaining resting BP and sympathetic tone [8]. Abnormalities in the function and structure of the RVLM are closely relative to pathophysiological precession of hypertension [11]. 
Notably, neuropathic pain also has an effect on neuronal activity in the RVLM. Jung et al. [12] reported that tooth pulpal pain elicited c-fos expression in cardiovascular centers, such as the nucleus tractus solitarius (NTS) and RVLM, and further moderated cardiovascular reflex function.

It is well known that glutamate, a major excitatory neurotransmitter, plays an important role in mediating cardiovascular regulation in the RVLM. Glutamate receptors including NMDA and AMPA receptors in the RVLM have been demonstrated to be involved in control of BP and cardiovascular reflexes $[8,11]$. Interestingly, an enhancement in tonically active glutamatergic inputs to the RVLM is reported to be responsible to hyperactivity of RVLM vasomotor neurons, high BP and sympathoexcitation in hypertension and heart failure $[13,14]$. Based on our $[15,16]$ and other's studies [17], the release of glutamate in the RVLM is increased in the spontaneous hypertensive rats (SHR). On the one hand, microinjection of the glutamate receptor (GluR) antagonist kynurenic acid (KYN) into the RVLM induces a significant decrease in resting BP in hypertensive rats but not in normotensive rats $[18,19]$. On the other hand, enhanced tonic glutamatergic input to the RVLM contributes to the hyperactivity of RVLM vasomotor neurons and sympathetic tone in rats with chronic heart failure [20]. Interestingly, neuropathic pain also enhances the release of the excitatory neurotransmitter glutamate in several brain regions including the thalamus, insular cortex, and periaqueductal gray (PAG) [21-23], which also send the fibers to connect with the RVLM. However, it is not clear whether high level of BP and sympathetic outflow induced by neuropathic pain is associated with an enhancement in tonically active glutamatergic inputs to the RVLM. Therefore, the hypothesis of the present study is that neuropathic pain enhances tonically active glutamatergic inputs to the RVLM, which contributes to high level of BP and sympathetic outflow. In order to test this hypothesis, we investigated the effects of neuropathic pain on the release of glutamate in the RVLM, expression of glutamate receptor NMDA, and the response of cardiovascular activity to RVLM injection of glutamate receptor antagonist.

\section{Materials and Methods}

2.1. Animals. Male Sprague-Dawley rats (220-260 g) were purchased from Sino-British SIPPR/BK Lab Animal Ltd. (Shanghai, China). Experimental protocols were approved by the Institutional Animal Care and Use Committees at Second Military Medical University and Shanghai JiaoTong University School of Medicine. All methods were performed in accordance with the relevant guidelines and regulations.

\subsection{Production of Animal Model with Neuropathic Pain. The} rat model with neuropathic pain was produced according to the previous study [24]. Briefly, rats were anesthetized with $10 \%$ chloral hydrate $(35 \mathrm{mg} / \mathrm{kg}$, ip). The skin below the bilateral eyes was shaved, and the position of the rat head was fixed. Below the eyes, a small, approximately $5 \mathrm{~mm}$, incision was made at the junction between the zygomatic arch and the nasal bone. Nerves were separated from the surrounding connective tissues carefully, and then the infraorbital nerve (ION), which is the secondary branch of the trigeminal nerve, was exposed clearly. Two absorbable threads (4-0 chromic catgut) were appropriately tied around the ION. The two ligatures were spaced approximately $2 \mathrm{~mm}$ apart from each other. After the surgery, the rats were fed in comfortable cages. The sham operation was performed by exposing the infraorbital nerve without ligatures.

2.3. Assessing Mechanical Sensitivity. To relieve stress during the study, the animals were trained to adapt to the environment and specific procedures one week before surgery. The behavioral tests were carried out with Von Frey filaments $[24,25]$. The baseline pain thresholds were measured 3 days prior to surgery and then measured again on postsurgery days $5,10,15,20,25$, and 30 . The mechanical stimulus intensities from low to high were $0.07 \mathrm{~g}, 0.16 \mathrm{~g}, 0.4 \mathrm{~g}, 0.6 \mathrm{~g}, 1.0 \mathrm{~g}$, $1.4 \mathrm{~g}, 2.0 \mathrm{~g}, 4.0 \mathrm{~g}, 6.0 \mathrm{~g}, 8.0 \mathrm{~g}, 10.0 \mathrm{~g}, 15.0 \mathrm{~g}$, and $26.0 \mathrm{~g}$. Each stimulus intensity was tested five times on the bilateral whisker pads of rats. The threshold value for mechanical pain was determined according to the corresponding stimulation strength to the rats presented with one or more of the following items: (1) Dodge actions, such as backward movement, turning around, or shaking the head; to avoid the stimuli, the rats curled their body, moved closer to the cage walls, or hid their face and head under their body; (2) scratching their face; the animals scratched the stimulated region on the face more than three times; and (3) aggressive behaviors; the rats grasped and bit the stimulating device and exhibited attack actions.

2.4. Measurement of Resting BP and HR. To determine whether the ION injury has an influence on cardiovascular activity, levels of $\mathrm{BP}$ and $\mathrm{HR}$ in conscious rats were recorded using a computerized noninvasive tail-cuff system (ALCNIBP, Shanghai Alcott Biotech) a week before and after the ION-CCI surgery. The measurement of $\mathrm{BP}$ and HR was carried out at days $5,10,15,20,25$, and 30 after the surgery. The measurement of BP and HR by tail-cuff method was performed according to our previous methods $[12,16]$. Briefly, the animals were placed in a specific holding device with a thermostatically controlled warming plate and then warmed to an ambient temperature of $32^{\circ} \mathrm{C}$ to keep the tail artery vasodilated. Approximately $20-30 \mathrm{~min}$ before recording the $\mathrm{BP}$, the rats were put in chambers to acclimate to the measuring chambers. Pressure was applied to the tail to occlude blood flow and was slowly released until the first pulse of arterial flow was detected. Then, the cuff was connected to a transducer, which could amplify the signal and record by a data acquisition system. At least six consecutive cycles were measured, and the averaged values were recorded.

2.5. In Vivo Experiments. The surgical procedures and recording of $\mathrm{BP}, \mathrm{HR}$, and renal sympathetic nerve activity (RSNA) under anesthetized condition were based on our previous studies $[20,26]$. Briefly, rats were anesthetized by urethane $(800 \mathrm{mg} / \mathrm{kg}$, ip) and achloralose $(40 \mathrm{mg} / \mathrm{kg}$, ip). A catheter was inserted into the right femoral artery to measure $\mathrm{BP}$, and the femoral vein was catheterized for supplemental 
drugs. Rats were placed on a stereotaxic frame, and head fixed horizontally, and dorsal surface of the medulla was surgically exposed. The renal sympathetic nerve was separated and recorded with a pair of recording electrodes. In order to avoid afferent activity, the distal terminus of the renal nerve was cut. Then, the RSNA signal was amplified and monitored together with BP and HR by the PowerLab system. The baseline RSNA was taken as $100 \%$ from the absolute value after the noise level was subtracted. The maximum RSNA was measured during euthanasia, as described previously [26]. Usually, the maximum nerve activity (Max) occurred 5 min after the rat was euthanized with an overdose of pentobarbital sodium $(200 \mathrm{mg} / \mathrm{kg})$. Background noise levels for sympathetic nerve activity were recorded 15-20 min after the rat was euthanized. According to the unit conversion of Powerlab Chart (AD Instruments) system, the Max value was set to $100 \%$, and the noise level was set to $0 \%$. Baseline nerve activity was taken as the percentage of Max. The body temperature was maintained at $37^{\circ} \mathrm{C}$ with a temperature controller.

2.6. RVLM Microinjection. As reported in our previous study [27], RVLM microinjection was performed with a threebarrel micropipette. The RVLM coordinates were 1.5$2.5 \mathrm{~mm}$ rostral to the obex, $1.8-2.0 \mathrm{~mm}$ lateral to the midline, and 3.0-3.2 mm deep to the dorsal surface of the brainstem. The microinjections were completed within $5-10 \mathrm{~s}$ by a pressure injector, and the microinjection volume was $100 \mathrm{nl}$. The functional location of the RVLM was identified by a pressor response to microinjection of L-glutamate $(1 \mathrm{nmol})$. The interval between bilateral injections was within $2 \mathrm{~min}$. The $\mathrm{BP}, \mathrm{HR}$, and RSNA were then recorded continuously for at least $60 \mathrm{~min}$ after the bilateral injection of $\mathrm{KYN}(2.7 \mathrm{nmol})$ into the RVLM. At the end of each experiment, a histological identification was conducted to verify microinjection site marked by dye blue.

2.7. Microdialysis. Brain microdialysis in vivo was carried out as previously described [28]. The rats were anesthetized with inhaled isoflurane (3\%), and the RVLM was surgically exposed as described above. A microdialysis probe (MAB.6.14.2) was inserted into the RVLM. Brain microdialysis was carried out by perfusing the probe with artificial cerebrospinal fluid at a rate of $2 \mu \mathrm{l} / \mathrm{min}$. The volume of each dialysate sample (10 min) was $20 \mu \mathrm{l}$, and the samples were obtained after at least $60 \mathrm{~min}$ of rest following the surgical operation.

2.8. Western Blot Analysis. Ten days after the ION-CCI surgery, the rat brains were obtained and stored at $-80^{\circ} \mathrm{C}$. The RVLM, PAG, and paraventricular nucleus (PVN) tissues were punched in accordance with the rat brain atlas [29]. In this study, the Obex point was suggested to a landmark to identify the RVLM, which is based on the brain alignment map, and then measured the location of each nucleus from this map. The freezing microtome was set the slice thickness of $50 \mu \mathrm{m}$, and the number of slices was counted to locate the longitudinal distance. The distance from RVLM to Obex is $2 \mathrm{~mm}$. To identify PVN, we located the anterior commissure based on the brain alignment map. The distance from the
PVN to the disappearing point of anterior commissure is $1.2 \mathrm{~mm}$. PAG is the area around the aqueduct of the brain, which was recognized in the transverse section of the brain. The lateral distance was measured according to the brain localization map, and the location of the nucleus from the midline of the brain was measured. Protein concentrations were measured using a BCA protein assay kit (Beyotime, Shanghai). The Western blot procedures were based on the previous study [25]. Equal amounts of protein $(40 \mu \mathrm{g})$ were separated by SDS-PAGE (10\% acrylamide) and then transferred to a polyvinylidene fluoride membrane. The membrane was blocked with 5\% nonfat milk dissolved in Tris-buffered saline solution containing $0.1 \%$ Tween 20 for $2 \mathrm{~h}$ at room temperature. Membranes were probed with primary antibody overnight at $4{ }^{\circ} \mathrm{C}$. The primary antibodies included anti-vGLUT2 $(1: 2000$, antivesicular glutamate transporter 2, MAB5504, EMD Millipore), anti-GLS2 (1:1000, antiglutaminase 2, AV43562; Sigma-Aldrich), and anti-NMDAR1(1:1000, ab2824-1, Abcam). $\beta$-Actin was used as a loading control. After washing the membranes three times for $5 \mathrm{~min}$ each, the membranes were incubated with a secondary antibody for $2 \mathrm{~h}$ at room temperature. After immunoblotting, the bands were visually detected and analyzed by the Syngene Bio Imaging system (number 55000, Gene Company).

2.9. High-Performance Liquid Chromatography (HPLC). The concentrations of glutamate in dialysis samples and the brain nuclei (PAG, PVN) were measured by HPLC as described previously [12]. After the PAG and PVN tissues were punched from the brain and weighed, $0.05 \mathrm{mM} \mathrm{HClO} 4$ was placed into the tube, and the tissues were homogenized and centrifuged for $10 \mathrm{~min}$, and the supernatant was collected for further analysis. There was no pretreatment for the dialysate samples from the RVLM. All the samples were analyzed by HPLC (model 582 pump, ESA) with electrochemical detection (model 5300, ESA). o-Phthalaldehyde (OPA)/2mercaptoethanol (b-ME) was used for derivatization for the amino acid analysis. Supernatant sample $(20 \mu \mathrm{l})$ was mixed with $50 \mu \mathrm{l}$ of OPA/b-ME solution. However, $50 \mu \mathrm{l}$ of the supernatant from the brain nuclei tissue samples was mixed with $20 \mu \mathrm{l}$ of OPA/b-ME solution and derivatized approximately $2 \mathrm{~min}$ before analysis, and then $50 \mu \mathrm{l}$ of the derivatized sample was injected for subsequent amino acid analysis. The HPLC analysis was carried out on a reversephase C18 column (Shiseido Capcell Pak $75 \times 3 \mathrm{~mm}, 3 \mu \mathrm{m}$ C18, P/N 88-90816, Shiseido Co. Ltd., Tokyo, Japan). The mobile phase consisted of $100 \mathrm{mM}$ anhydrous disodium hydrogen phosphate, $22 \%$ methanol, and $3.5 \%$ acetonitrile at $\mathrm{pH} 6.75$, and the flow rate was $0.5 \mathrm{ml} / \mathrm{min}$. The detection channel potentials were set at $+150 \mathrm{mV}$ and $+550 \mathrm{mV}$.

Content of norepinephrine (NE) in $24 \mathrm{~h}$ urine was also detected by HPLC. Twenty-four-hour urinary samples were collected from metabolism cages in which the rats were placed for $24 \mathrm{~h}$. To reduce the degradation of NE, the samples were acidified with glacial acetic acid in $15 \mathrm{ml}$ centrifuge tubes, which were embedded in crushed ice. Dihydroxybenzylamine (Sigma) was used as the internal standard. Before the experiment, the samples were dissociated carefully 
according to the detailed procedure referenced in our previous report [16]. The flow rate was $0.5 \mathrm{ml} / \mathrm{min}$. The HPLC data were acquired, processed, and analyzed using Coulochem software.

2.10. Immunohistochemistry. The immunohistochemistry procedures were carried out according to our previous study [30]. The rats were killed with an overdose of sodium pentobarbital $(200 \mathrm{mg} / \mathrm{kg}$, ip) and perfused with $0.9 \%$ saline followed by $4 \%$ paraformaldehyde. The brains were dissected, postfixed with $4 \%$ paraformaldehyde for approximately $24 \mathrm{~h}$, and then cryoprotected in $20 \%$ sucrose for at least $24 \mathrm{~h}$. Brain sections were made with a freezing microtome (Leica, CM1850), and the thickness of sections was $10 \mu \mathrm{m}$. The free-floating sections were rinsed 3 times with $0.1 \mathrm{M} \mathrm{PBS}$ and blocked with 5\% BSA for $1 \mathrm{~h}$ at $37^{\circ} \mathrm{C}$ immediately prior to the incubation with primary antibody. The sections were incubated with the primary antibodies overnight at $4^{\circ} \mathrm{C}$. The primary antibody was rabbit anti-fos (1:100; Phoenix Pharmaceuticals Inc., Burlingame, CA, USA). For direct staining, the sections were washed in $0.1 \mathrm{M}$ PBS 3 times at $5 \mathrm{~min}$ intervals and incubated with secondary antibody for $2 \mathrm{~h}$ at room temperature. Finally, the diaminobenzidine $(\mathrm{DAB})$ coloration method was used to determine the expression of $\mathrm{c}$-fos protein.

2.11. Data Analysis. Values are presented as the means \pm SD. Statistical analyses were carried out using SPSS software (version 15.0). To assess the effect of the ION-CCI surgery on mechanical hypersensitivity, a repeated measures analysis of variance (ANOVA) followed by a post hoc Bonferroni test was applied to evaluate the mechanical threshold before and after surgery. The values of BP obtained by tail-cuff method were also analyzed and compared using a repeated measures ANOVA. The extent of the glutamate in the RVLM and BP during different time courses was compared using a Factorial design ANOVA followed by a post-LSD test. Other data were analyzed using unpaired $t$ tests. Differences were considered to be significant at $p<0.05$.

\section{Results}

3.1. Effects of ION-CCI on Hyperalgesia and Resting BP. The mechanical thresholds after ION-CCI were measured repeatedly to confirm that the pain influence was continuous. We found that the basal mechanical thresholds (before surgery) between the sham and ION-CCI groups were not significantly different $(9.64 \pm 1.58$ versus $9.68 \pm 0.79 \mathrm{~g}, p>0.05)$. However, mechanical threshold of the ION-CCI group was decreased significantly 10 days after the ION-CCI surgery and persisted to 30 days after operation (Figure 1). As shown in Figure 2(a), levels of mean arterial pressure (MAP) from 5-day postoperation (PO5) $(158 \pm 9 \mathrm{mmHg})$ to PO20 $(140 \pm 8 \mathrm{mmHg})$ were significantly higher in the ION-CCI rats than in the sham rats. However, a maximal increase in MAP reached on PO10 in the ION-CCI rats $(169 \pm 10 \mathrm{mmHg})$. Ten days after the surgery, these two groups were anesthetized and MAP was measured through the arterial cannula. It was found that MAP was also

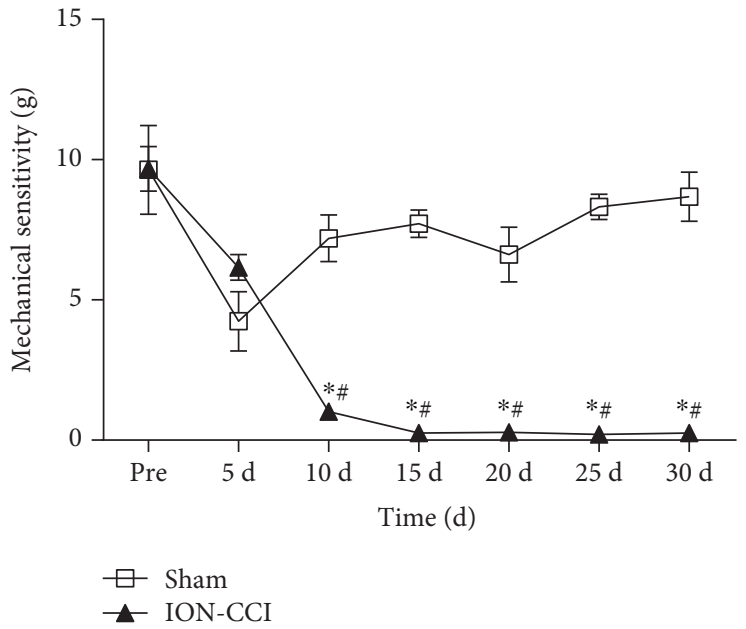

FIgURE 1: Time courses of the mechanical sensitivity in sham and ION-CCI rats. The data of PRE indicates an average of mechanical sensitivity at 1 and 2 days before surgery. $n=5$ /group. ${ }^{*} p<0.05$ compared with PRE value, ${ }^{\#} p<0.05$ compared with sham.

significantly higher $(146 \pm 8$ versus $109 \pm 7 \mathrm{mmHg}$ ) in the ION-CCI rats than in the sham rats (Figure 2(b)).

3.2. Effects of ION-CCI on $24 \mathrm{~h}$ Urinary Excretion of NE and RSNA. To identify whether trigeminal neuropathic pain increases sympathetic nerve activity, the $24 \mathrm{~h}$ urinary excretion of NE and RSNA was measured 10 days after the ION$\mathrm{CCI}$ and sham operation. As illustrated in Figures 3(a) and 3 (b), the $24 \mathrm{~h}$ urinary excretion of NE was increased significantly in the ION-CCI group compared with the sham group $(0.32 \pm 0.08$ versus $0.15 \pm 0.04 \mu \mathrm{g}, n=5, p<0.05)$. Similar to the NE result, the baseline RSNA (of Max) in the ION-CCI group was predominantly higher than that in the sham group $(29.5 \pm 3.7$ versus $13.2 \pm 2.2 \%, n=5, p<0.05)$.

3.3. Effects of ION-CCI on the Release of Glutamate in the $R V L M$. Figure 4 shows glutamate concentration in the microdialysis fluid of the RVLM. The glutamate content was significantly higher in the ION-CCI group compared with the sham rats on 10 days $(1636 \pm 169$ versus $347 \pm$ $24 \mu \mathrm{g} / \mathrm{l}), 15$ days $(1147 \pm 149$ versus $309 \pm 31 \mu \mathrm{g} / \mathrm{l}), 20$ days ( $757 \pm 53$ versus $405 \pm 79 \mu \mathrm{g} / \mathrm{l})$, and 25 days ( $470 \pm 43$ versus $367 \pm 58 \mu \mathrm{g} / \mathrm{l})$ after ION-CCI operation. The glutamate content began to increase at 5 days, reached the peak value on 10 days, and persisted on 30 days postoperation. However, the glutamate concentration in the RVLM of the sham group was no different at the different time points. Moreover, the level of NMDAR1 protein expression on RVLM in the ION-CCI group was higher than that in the sham group (Figure 4(b)).

3.4. Cardiovascular Effects of Bilateral Microinjections of $K Y N$ into the RVLM. Figure 5(a) represents original tracings of BP, HR, and RSNA in response to microinjection of KYN ( $2.7 \mathrm{nmol}$ for each side) into the RVLM. Bilateral injection of KYN into the RVLM produced a significant decrease in MAP $(-20.0 \pm 5.4 \%)$, HR $(-15.7 \pm 5.5 \%)$, and 


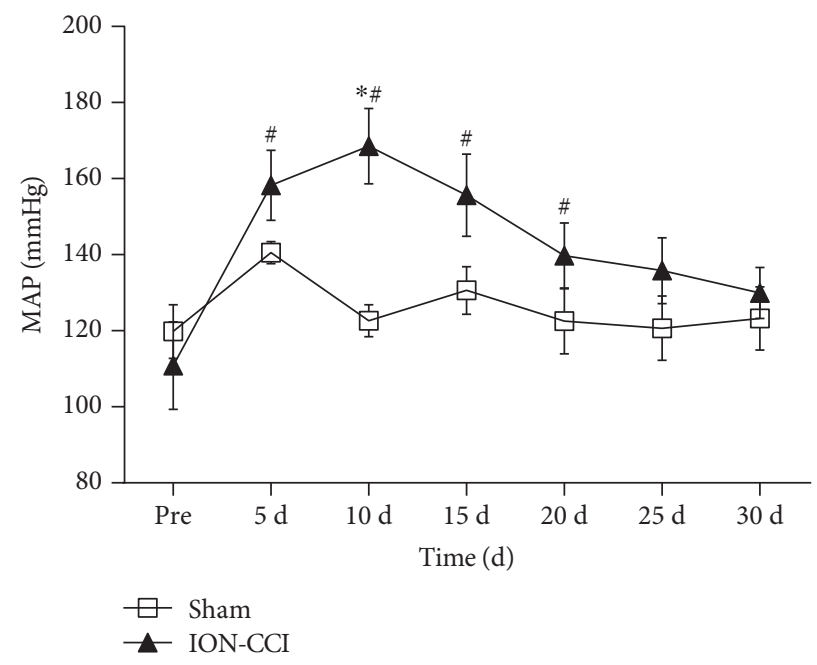

(a)

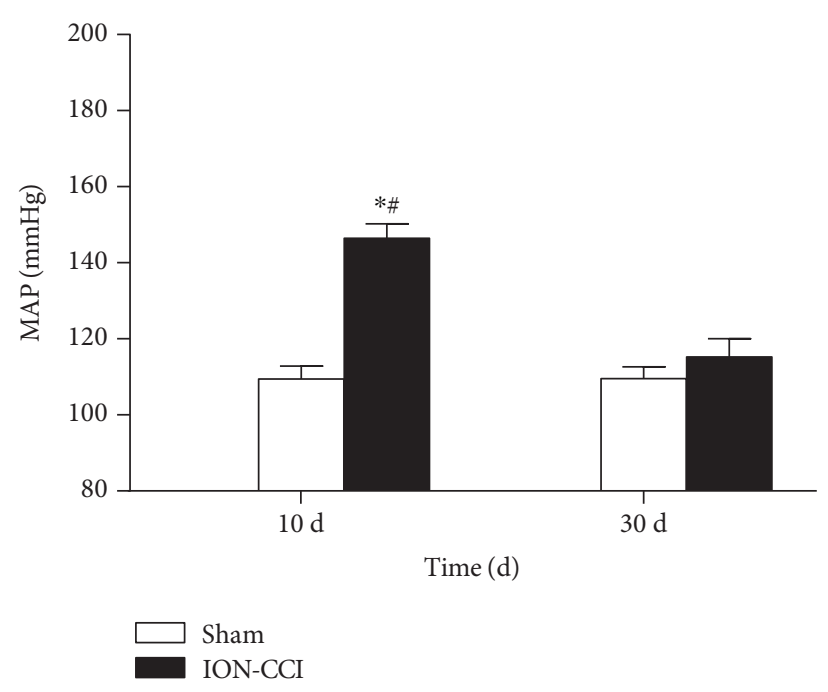

(b)

FIGURE 2: Effects of ION-CCI on blood pressure. (a) Time course of mean arterial pressure (MAP) consciously measured by tail-cuff method in awake rats. $n=5$ /group, ${ }^{*} p<0.05$ compared with PRE, ${ }^{*} p<0.05$ compared with sham. (b) MAP measured through the arterial cannula in anesthetized rats. $n=5$ /group ${ }^{*} p<0.05$ compared with $10 \mathrm{~d},{ }^{*} p<0.05$ compared with sham.

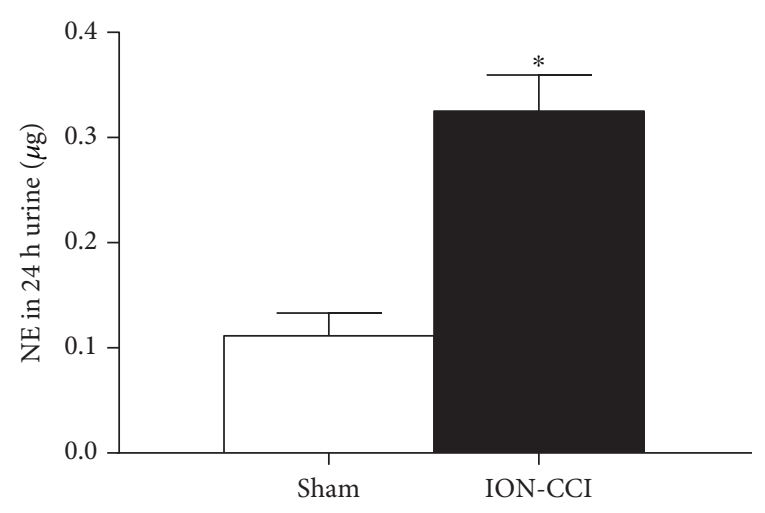

(a)

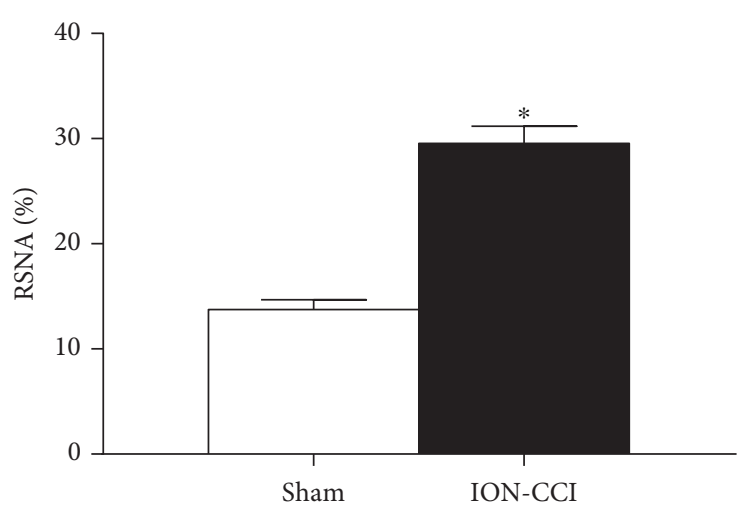

(b)

FIGURE 3: Effects of ION-CCI on basal renal sympathetic nerve activity (RSNA, (b)) and increased the $24 \mathrm{~h}$ urinary excretion of norepinephrine (NE, (a)). $n=7$ in each group. ${ }^{*} p<0.05$ compared with sham.

RSNA $(-23.5 \pm 6.7 \%)$ in the ION-CCI rats but not in the sham rats (Figure $5(\mathrm{~b})$ ).

\subsection{Effects of ION-CCI on the Glutamate Content and the} Expression of vGLUT2 and Glutaminase 2 (GLS2) in the $P A G$ and $P V N$. As depicted in Figure 6, the glutamate concentration in the PAG and PVN was significantly higher on 10 days after ION-CCI. The levels of GLS2 and vGLUT2 protein expressions in the PAG and PVN were also significantly increased in the ION-CCI rats compared with the sham rats.

3.6. Effects of ION-CCI on c-Fos Expression in the PAG, PVN, and RVLM. In this study, we used Fos-positive cells to examine the activity of neurons. The ION-CCI rats showed a significant increase in Fos expression in the PAG, PVN, and RVLM (Figure 7). The number of Fos-positive cells in the PAG and RVLM was increased by approximately 3-4 times in the ION-CCI group more than in the sham group. The number of Fos-positive neurons in the PVN of the ION-CCI rats was much greater than that in the PVN of the sham group.

\section{Discussion}

The major findings of our present experiments are the following: (1) ION-CCI effectively increased BP and sympathetic nerve activity; (2) ION-CCI significantly increased the tonic release of glutamate in the RVLM; and (3) the PAG and PVN may be important sources for enhanced tonic glutamatergic inputs to the RVLM in the ION-CCI. Based on these results, it is suggested that the enhancement of tonically active glutamatergic inputs to the RVLM plays an important role in the neuropathic pain associated with increase in $\mathrm{BP}$ and sympathetic nerve activity. 


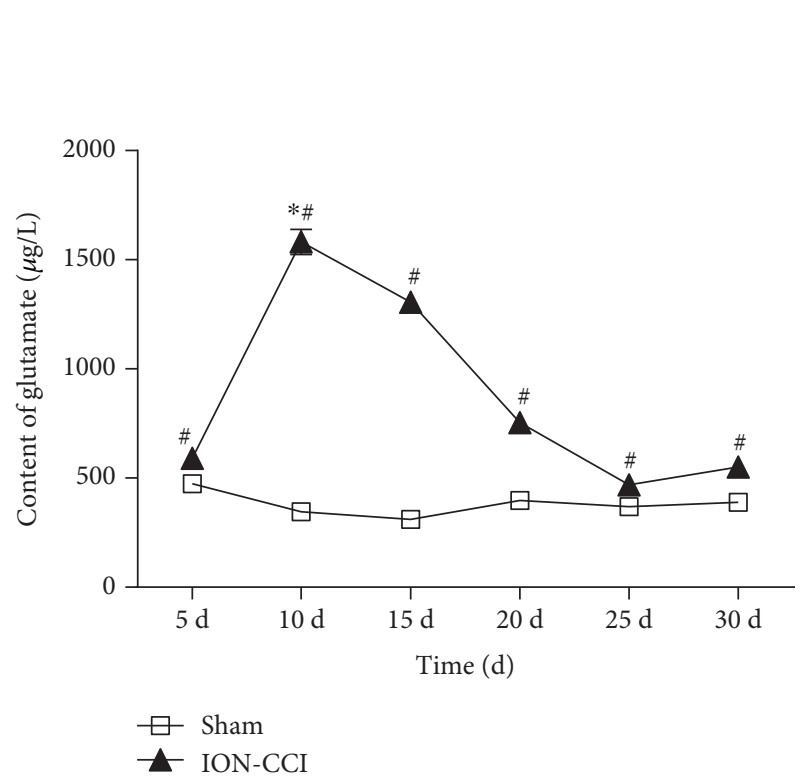

(a)

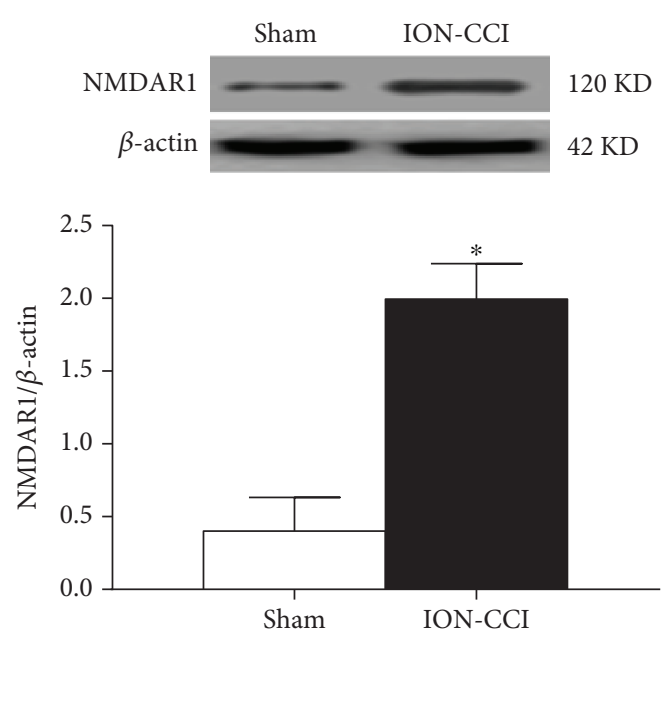

(b)

FIgURE 4: Effects of ION-CCI on glutamate release and glutamate receptor expression in the RVLM. (a) Time course of content of glutamate in dialysis fluid from the RVLM in the ION-CCI group. $n=4$ /group. ${ }^{*} p<0.05$ compared with PO5, ${ }^{\#} p<0.05$ compared with sham. (b) NMDAR1 expression levels in the RVLM. $n=5$ /group. ${ }^{*} p<0.05$ compared with sham.

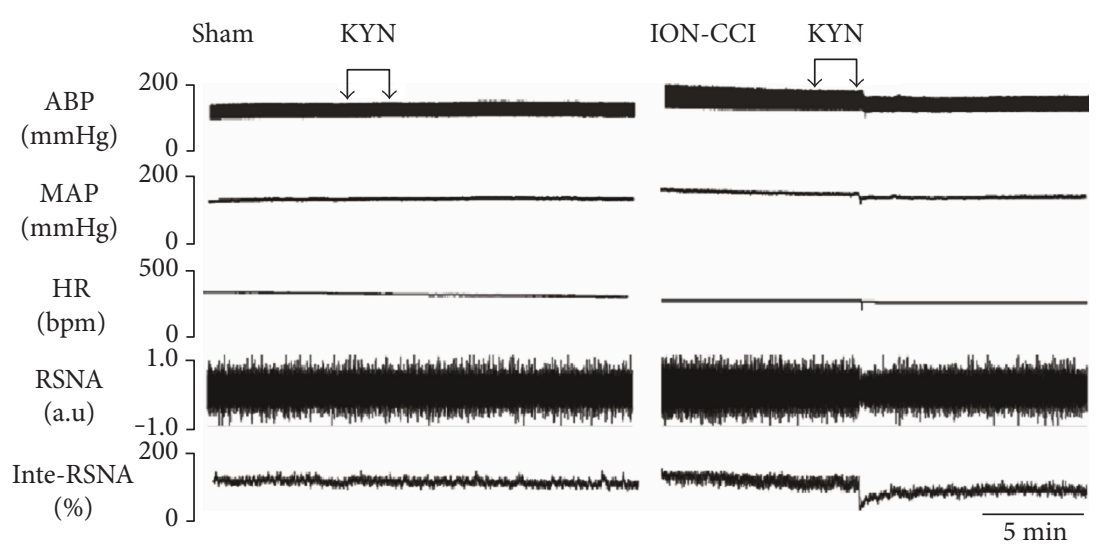

(a)

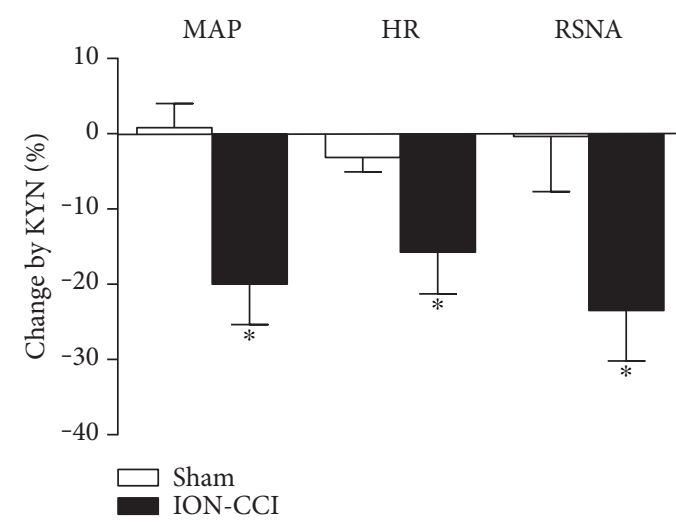

(b)

FIgURE 5: Cardiovascular response to microinjection of KYN into the RVLM in the ION-CCI group. Representative tracings (a) and percent changes (b) of BP, HR, and RSNA in response to microinjection of KYN into the RVLM in the sham and ION-CCI groups. $n=5 /$ group, ${ }^{*} p<0.05$ compared with sham. 

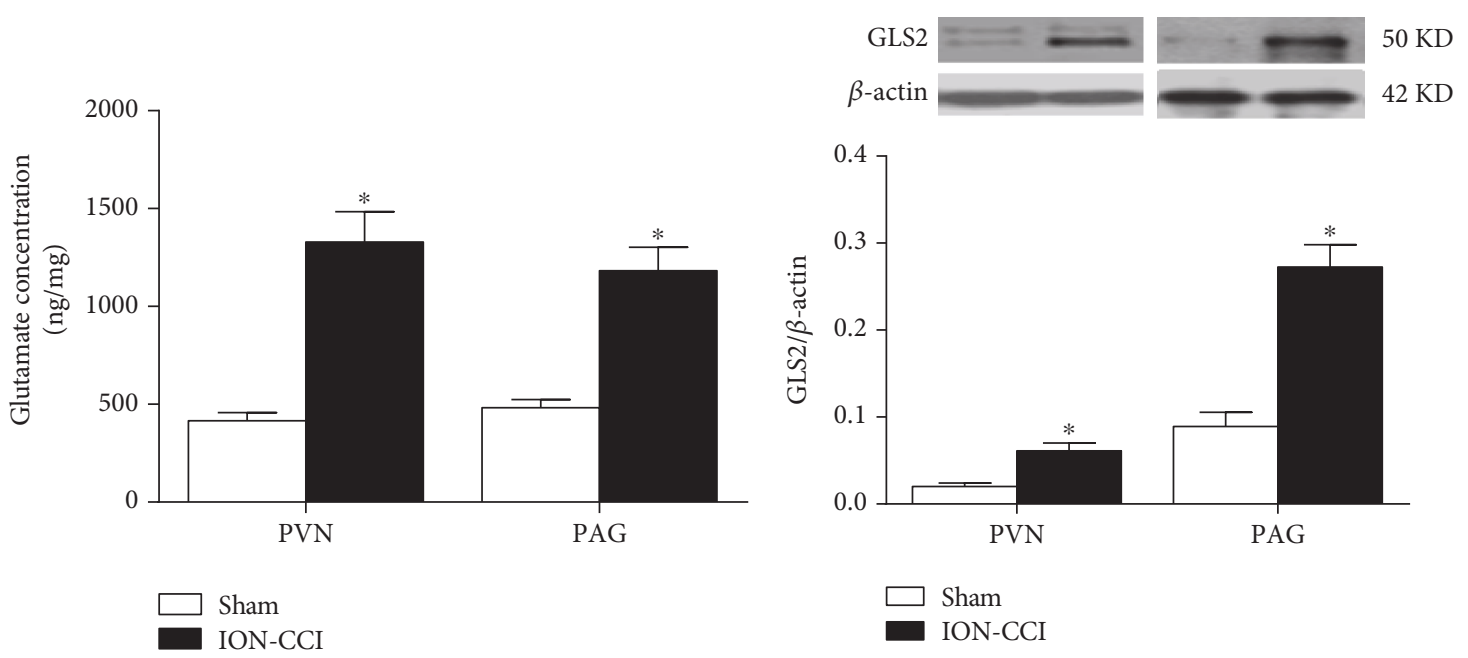

(a)

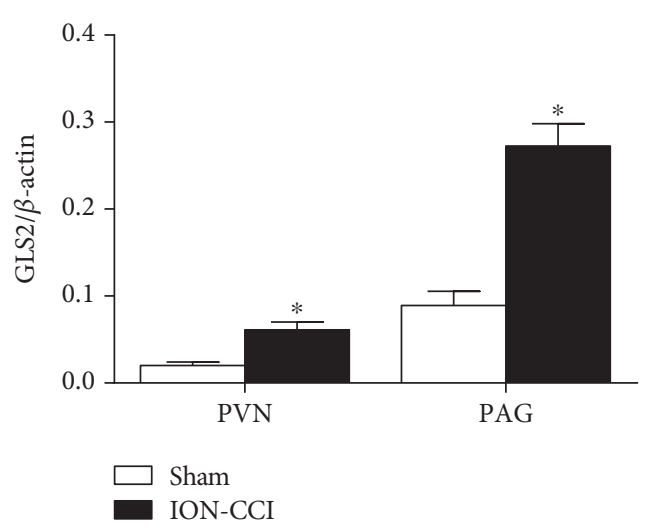

(b)

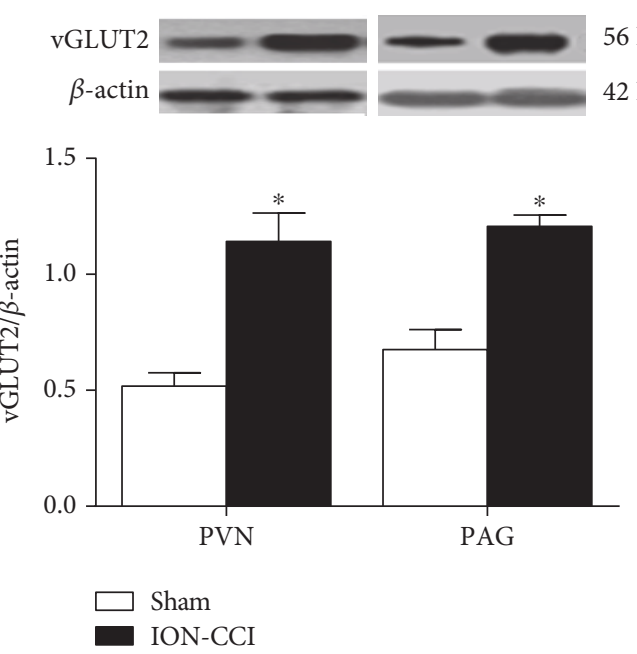

(c)

FIGURE 6: Effects of ION-CCI on the content of glutamate and the expression of vGLUT2 and GLS2 proteins in the PAG and PVN. (a) The concentration of glutamate and the protein levels of GLS2 and vGLUT2 in the PAG (a) and PVN (b), $n=5 /$ group. ${ }^{*} p<0.05$ compared with sham.

In this study, VonFrey filaments were used to evaluate the CCI. It is confirmed that ION-CCI significantly decreases the mechanical pain threshold in the trigeminal nerve's innervating regions, which leads to neuropathic pain syndromes [31]. Additionally, we determined the mechanical pain threshold for 30 days and obtained the reduplicated value of the MAP in conscious rats, which was an abrupt increase of MAP 10 days after the ION-CCI surgery. These observations are similar to the previous evidence in sciatic nerve CCI, in which basal $\mathrm{BP}$ of the rats remains a high level during the first 2 weeks after the CCI [32]. It was also observed that the BP level measured by the femoral artery in anesthetized rats was significantly increased in the ION-CCI rats. We found that the level of BP measured by the tail-cuff method in awake rats was somewhat higher than the BP that was directly obtained from the femoral artery in anesthetized rats. This is possible that stress could result in a rise in BP. One of the most important mechanisms of hypertension is the overactivity of SNS. Partial nerve injury or tissue damage results in a change in peripheral nociceptors, which is associated with overactivity of the SNS [33]. In the present study, we measured the RSNA and $24 \mathrm{~h}$ urinary excretion of NE on PO10 and found that the RSNA and $24 \mathrm{~h}$ urinary excretion of NE were higher in the ION-CCI rats compared with the sham rats. These results indicate that the trigeminal neuropathic pain increases sympathetic nerve activity. Collectively, these data suggested that trigeminal neuropathic pain could substantially increase BP and sympathetic activity.

Pain, a type of noxious stimulation, has been demonstrated to result in an increase in the release of excitatory neurotransmitters (e.g., glutamate). Many studies have found that pain can lead to the increased release of glutamate in certain brain areas, such as the PAG [34], RVM [35], and insular cortex [21]. Our previous research $[12,27]$ has reported that tonically active glutamatergic input to the RVLM is significantly enhanced in SHRs and heart failure. In this study, we measured the glutamate concentration in the RVLM microdialysis samples over different time courses. 


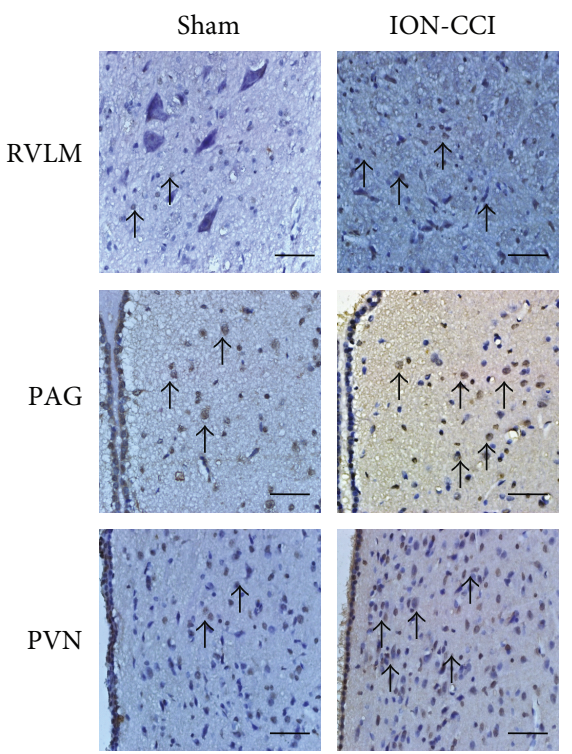

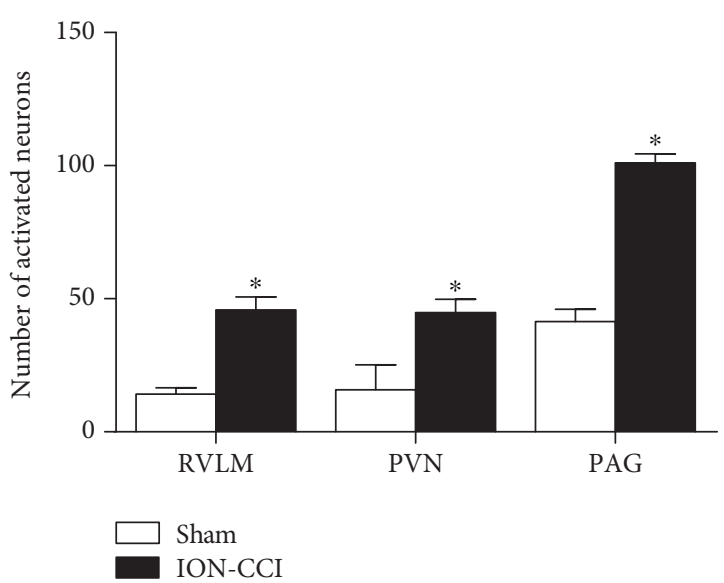

(b)

FIGURE 7: Effects of ION-CCI on the expressions of c-fos protein in the RVLM, PAG, and PVN. Immunostaining images (a) and group data (b) of fos-positive cells in the RVLM, PAG, and PVN in the sham and ION-CCI groups. The scale bar is $50 \mu \mathrm{m}$ in (a). $n=5 /$ group. ${ }^{*} p<0.05$ compared with sham.

The results indicate that the glutamate concentration in the ION-CCI group was obviously higher compared with the sham group. The glutamate concentration in the ION-CCI group reached a peak on PO10, which is consistent with change in BP. Furthermore, microinjection of the glutamate receptor antagonist KYN into the RVLM induced a significant reduction in BP, HR, and RSNA in the ION-CCI group but not in the sham rats. These data suggest that the trigeminal neuropathic pain enhances the tonically active glutamatergic inputs to the RVLM. It is suggested that KYN, a nonselective receptor antagonist, blocks both NMDA and AMPA receptors. NMDA and AMPA receptors are two subtypes of ionotropic glutamate receptors that play an important role in cardiovascular control and the occurrence of neuropathic pain $[36,37]$. Our study focused on the presynaptic release of glutamate, which may activate both NMDA and AMPA receptors. So we selected KYN to block both NMDA and AMPA receptors. However, we do not exclude the possibility that NMDA and AMPA receptors may have different roles in mediating the effects of IONCCI-induced release of glutamate. In addition, the present study was focused on the cardiovascular effects in response to neuropathic pain. KYN was injected acutely in the anesthetized state, and it may be difficult to measure the pain threshold again after injection. It is not clear whether blocking glutamatergic activity in RVLM is able to normalize the reduction of mechanical sensitivity (pain threshold).

Glutamate release is mainly dependent on synthesis of glutamate and nerve impulses in the presynaptic mechanism. The catalytic synthesis of glutamate-releasing glutaminase and neuronal excitability was subjected to determine the intensity level of glutamatergic input to the RVLM $[38,39]$. It has been indicated that the glutamatergic inputs to the RVLM originate from various areas, such as the PAG and PVN $[40,41]$. Functional changes in these areas have a direct effect on the RVLM sympathetic output and its control of cardiovascular function. The RVLM has some connections with the PAG and PVN, which has an effect on the regulation of cardiovascular activity [34, 42-44]. In this study, we found that the glutamate content in the PAG and PVN was also increased in the trigeminal neuropathic pain rats. Furthermore, we observed that trigeminal neuropathic pain led to a significant augmentation in the expression of GLS2, a key enzyme for glutamate synthesis, in the PAG and PVN. The protein level of vGLUT2, which can package the glutamate into presynaptic vesicles so that these vesicles can be released into the synapse, was also increased in the trigeminal neuropathic pain rats. A previous study from our laboratory suggested that exercise training resulted in a significant decrease in the expression level of GLS2 in the PVN of SHR [12]. Glutamatergic inputs to the RVLM may originate from multiple sources, including the above-mentioned regions within the brainstem and forebrain. The key mechanism of enhanced tonic glutamatergic inputs to RVLM is presynaptic release of glutamate, which is based on neurotransmitter synthesis and nerve impulse. A limitation of this study is that we do not further determine the changes of afferent nerve impulses to the RVLM by electrophysiological approach.

The RVLM plays an important role in maintaining resting $\mathrm{BP}$ and sympathetic tone, and sympathetic nerve 
activity is regulated by the activities of RVLM neurons. The c-Fos protein is a product of the c-fos gene, which is commonly used as a marker of changes in neuronal activity [45]. Pain can cause a strong enhancement of c-Fos expression in several brain regions [46]. The presented data revealed that trigeminal neuropathic pain provoked c-Fos expression in the RVLM of the ION-CCI rats, suggesting that trigeminal neuropathic pain is an inducer or stressor of RVLM neurons. Trigeminal neuropathic pain causes the excitation of RVLM neurons and leads to the excitation of sympathetic nerves. Furthermore, we also confirmed that c-Fos expression in the PVN and PAG was also increased in the ION-CCI rats. PVN and RVLM have many kinds of neurons, all of which can express c-fos. In this work, we did not identify neurons for c-fos expression, which is a limitation of our study. A significant change in cardiovascular activity in response to injection of RVLM KYN was confirmed, suggesting that the autonomic/presympathetic neurons in the RVLM are involved in the processing of ION-CCI. In addition, neuropathic pain is a kind of chronic noxious stimulation. It is suggested that many nuclei in the brain may be affected in response to neuropathic pain. We do not rule out the involvement of other regions such as cortex and hippocampus.

Our present study has demonstrated that trigeminal neuropathic pain increased BP and sympathetic outflow, which is associated with enhancement in tonically active glutamatergic inputs to the RVLM. This mechanism may contribute to autonomic regulation of cardiovascular activity under the state of neuropathic pain.

\section{Conflicts of Interest}

The authors declare that they have no conflicts of interest.

\section{Authors' Contributions}

Wei Wang, Zui Zou, and Xing Tan contributed equally to this work.

\section{Acknowledgments}

The authors gratefully acknowledge the statistical assistance of Dr Jian Lu (from the Department of Statistical Analysis, SMMU). This work was supported by the National Natural Science Foundation of China (nos. 81370363, 81570385, and 81630012 to Wei-Zhong Wang; 81372013 to Xue-Yin Shi), the SMMU Creativity and Innovation Training Program (2014QN02 to Ru-Wen Zhang), and the 12th Five-Year Plan of the People's Liberation Army Key Project Grant (BWS12J027 to Xue-Yin Shi).

\section{References}

[1] N. Attal, A. Masselin-Dubois, V. Martinez et al., "Does cognitive functioning predict chronic pain? Results from a prospective surgical cohort," Brain, vol. 137, pp. 904-917, 2014.

[2] N. Attal, M. Lanteri-Minet, B. Laurent, J. Fermanian, and D. Bouhassira, "The specific disease burden of neuropathic pain: results of a French nationwide survey," Pain, vol. 152, no. 12, pp. 2836-2843, 2011.

[3] S. Bruehl, O. Y. Chung, J. N. Jirjis, and S. Biridepalli, "Prevalence of clinical hypertension in patients with chronic pain compared to nonpain general medical patients," The Clinical Journal of Pain, vol. 21, no. 2, pp. 147-153, 2005.

[4] R. B. Olsen, S. Bruehl, C. S. Nielsen, L. A. Rosseland, A. E. Eggen, and A. Stubhaug, "Hypertension prevalence and diminished blood pressure-related hypoalgesia in individuals reporting chronic pain in a general population. The Troms $\varnothing$ Study," Pain, vol. 154, no. 2, pp. 257-262, 2012.

[5] S.-L. Pan, L.-S. Chen, M.-F. Yen, Y.-H. Chiu, and H.-H. Chen, "Increased risk of stroke after trigeminal neuralgia-a population-based follow-up study," Cephalalgia, vol. 31, no. 8, pp. 937-942, 2011.

[6] N. J. Goodson, B. H. Smith, L. J. Hocking et al., "Cardiovascular risk factors associated with the metabolic syndrome are more prevalent in people reporting chronic pain: results from a cross-sectional general population study," Pain, vol. 154, no. 9, pp. 1595-1602, 2013.

[7] A. Teruel, S. Ram, S. K. Kumar, S. Hariri, and G. T. Clark, "Prevalence of hypertension in patients with trigeminal neuralgia," Journal of Headache and Pain, vol. 10, no. 3, pp. 199-201, 2009.

[8] S. C. Malpas, "Sympathetic nervous system overactivity and its role in the development of cardiovascular disease," Physiological Reviews, vol. 90, no. 2, pp. 513-557, 2010.

[9] P. G. Guyenet, "The sympathetic control of blood pressure," Nature Reviews Neuroscience, vol. 7, no. 5, pp. 335-346, 2006.

[10] T. Schlereth and F. Birklein, "The sympathetic nervous system and pain," Neuromolecular Medicine, vol. 10, no. 3, pp. 141147, 2008.

[11] A. F. Sved, S. Ito, and J. C. Sved, "Brainstem mechanisms of hypertension: role of the rostral ventrolateral medulla," Current Hypertension Reports, vol. 5, no. 3, pp. 262-268, 2003.

[12] J.-Y. Jung, H.-R. Yang, Y.-J. Jeong et al., "Effects of acupuncture on c-Fos expression in brain after noxious tooth stimulation of the rat," The American Journal of Chinese Medicine, vol. 34, no. 06, pp. 989-1003, 2006.

[13] Z. Yang, D. Bertram, and J. H. Coote, "The role of glutamate and vasopressin in the excitation of RVL neurones by paraventricular neurones," Brain Research, vol. 908, no. 1, pp. 99-103, 2001.

[14] M. K. Sun and D. J. Reis, "NMDA receptor-mediated sympathetic chemoreflex excitation of RVL-spinal vasomotor neurones in rats," Journal of Physiology, vol. 482, Part 1, pp. 53-68, 1995.

[15] Y. P. Zha, Y. K. Wang, Y. Deng et al., "Exercise training lowers the enhanced tonically active glutamatergic input to the rostral ventrolateral medulla in hypertensive rats," CNS Neuroscience \& Therapeutics, vol. 19, no. 4, pp. 244-251, 2013.

[16] Y. K. Wang, D. Shen, Q. Hao et al., "Overexpression of angiotensin-converting enzyme 2 attenuates tonically active glutamatergic input to the rostral ventrolateral medulla in hypertensive rats," American Journal of Physiology-Heart and Circulatory Physiology, vol. 307, no. 2, pp. H182-H190, 2014.

[17] T. Kubo, M. Kihara, and Y. Misu, "Genetically altered brain amino acid metabolism in spontaneously hypertensive rats: a study by using young spontaneously hypertensive rats and renal hypertensive rats," Clinical and Experimental Hypertension, vol. 12, no. 7, pp. 1191-1201, 1990. 
[18] S. Ito, K. Komatsu, K. Tsukamoto, and A. F. Sved, "Excitatory amino acids in the rostral ventrolateral medulla support blood pressure in spontaneously hypertensive rats," Hypertension, vol. 35, no. 1, Part 2, pp. 413-417, 2000.

[19] S. Ito, K. Komatsu, K. Tsukamoto, and A. F. Sved, "Tonic excitatory input to the rostral ventrolateral medulla in Dahl salt-sensitive rats," Hypertension, vol. 37, no. 2, Part 2, pp. 687-691, 2001.

[20] W. Z. Wang, L. Gao, H. J. Wang, I. H. Zucker, and W. Wang, "Tonic glutamatergic input in the rostral ventrolateral medulla is increased in rats with chronic heart failure," Hypertension, vol. 53, no. 2, pp. 370-374, 2009.

[21] S. Qiu, T. Chen, K. Koga et al., "An increase in synaptic NMDA receptors in the insular cortex contributes to neuropathic pain," Science Signaling, vol. 6, no. 275, article ra34, 2013.

[22] L. F. Da Silva, J. M. Desantana, and K. A. Sluka, "Activation of NMDA receptors in the brainstem, rostral ventromedial medulla, and nucleus reticularis gigantocellularis mediates mechanical hyperalgesia produced by repeated intramuscular injections of acidic saline in rats," The Journal of Pain, vol. 11, no. 4, pp. 378-387, 2010.

[23] R. Radhakrishnan and K. A. Sluka, "Increased glutamate and decreased glycine release in the rostral ventromedial medulla during induction of a pre-clinical model of chronic widespread muscle pain," Neuroscience Letters, vol. 457, no. 3, pp. 141$145,2009$.

[24] M. Kernisant, R. W. Gear, L. Jasmin, J.-P. Vit, and P. T. Ohara, "Chronic constriction injury of the infraorbital nerve in the rat using modified syringe needle," Journal of Neuroscience Methods, vol. 172, no. 1, pp. 43-47, 2008.

[25] M. Cha, K. J. Kohan, X. Zuo, J. X. Ling, and J. G. Gu, "Assessment of chronic trigeminal neuropathic pain by the orofacial operant test in rats," Behavioural Brain Research, vol. 234, no. 1, pp. 82-90, 2012.

[26] J. Peng, Y.-K. Wang, L.-G. Wang et al., "Sympathoinhibitory mechanism of moxonidine: role of the inducible nitric oxide synthase in the rostral ventrolateral medulla," Cardiovascular Research, vol. 84, no. 2, pp. 283-291, 2009.

[27] Y. K. Wang, Q. Yu, X. Tan et al., "Centrally acting drug moxonidine decreases reactive oxygen species via inactivation of the phosphoinositide-3 kinase signaling in the rostral ventrolateral medulla in hypertensive rats," Journal of Hypertension, vol. 34, no. 5, pp. 993-1004, 2016.

[28] J. F. Peng, Z. T. Wu, Y. K. Wang et al., "GABAergic mechanism in the rostral ventrolateral medulla contributes to the hypotension of moxonidine," Cardiovascular Research, vol. 89, no. 2, pp. 473-483, 2010.

[29] G. Paxinos and C. Watson, The Rat Brain in Stereotaxic Coordinates, Academic, New York, 3rd edition, 1998.

[30] J. W. Maniscalco, H. Zheng, P. J. Gordon, and L. Rinaman, "Negative energy balance blocks neural and behavioral responses to acute stress by "silencing" Central glucagon-like peptide 1 signaling in rats," Journal of Neuroscience, vol. 35, no. 30, pp. 10701-10714, 2015.

[31] J. Gutierrez, S. Raju, J. P. Riley, and N. M. Boulis, "Introduction to neuropathic pain syndromes," Neurosurgery Clinics of North America, vol. 25, no. 4, pp. 639-662, 2014.

[32] Y. Jin, J. Sato, M. Yamazaki et al., "Changes in cardiovascular parameters and plasma norepinephrine level in rats after chronic constriction injury on the sciatic nerve," Pain, vol. 135, no. 3, pp. 221-231, 2008.
[33] T. Kawada, T. Akiyama, S. Shimizu et al., "Acute effects of arterial baroreflex on sympathetic nerve activity and plasma norepinephrine concentration," Autonomic NeuroscienceBasic \& Clinical, vol. 186, pp. 62-68, 2014.

[34] M. Rodríguez-Muñoz, P. Sánchez-Blázquez, A. VicenteSánchez, E. Berrocoso, and J. Garzón, "The mu-opioid receptor and the NMDA receptor associate in PAG neurons: implications in pain control," Neuropsychopharmacology, vol. 37, no. 2, pp. 338-349, 2011.

[35] L. F. S. D. Silva, R. Y. Walder, B. L. Davidson, S. P. Wilson, and K. A. Sluka, "Changes in expression of NMDA-NR1 receptor subunits in the rostral ventromedial medulla modulate pain behaviors," Pain, vol. 151, no. 1, pp. 155-161, 2010.

[36] S. Y. Chen, W. C. Wu, C. J. Tseng, J. S. Kuo, and C. Y. Chai, "Involvement of non-NMDA and NMDA receptors in glutamate-induced pressor or depressor responses of the pons and medulla," Clinical \& Experimental Pharmacology \& Physiology, vol. 24, no. 1, pp. 46-56, 1997.

[37] E. Kondo, K. Iwata, A. Ogawa et al., "Involvement of glutamate receptors on hyperexcitability of wide dynamic range neurons in the gracile nucleus of the rats with experimental mononeuropathy," Pain, vol. 95, no. 1, pp. 153-163, 2002.

[38] A. F. Sved, "Tonic glutamatergic drive of RVLM vasomotor neurons?," American Journal of Physiology-Regulatory Integrative and Comparative Physiology, vol. 287, no. 6, pp. R1301R1303, 2004.

[39] D. Soto, X. Altafaj, C. Sindreu, and A. Bayes, "Glutamate receptor mutations in psychiatric and neurodevelopmental disorders," Communicative \& Integrative Biology journal, vol. 7, no. 1, article e27887, 2014.

[40] H. Ferreira-Neto, S. Yao, and V. Antunes, "Purinergic and glutamatergic interactions in the hypothalamic paraventricular nucleus modulate sympathetic outflow," Purinergic Signalling, vol. 9, no. 3, pp. 337-349, 2013.

[41] G. G. Pelosi, C. Busnardo, R. F. Tavares, and F. M. A. Corrêa, "Cardiovascular responses to glutamate microinjection in the dorsomedial periaqueductal gray of unanesthetized rats," Journal of Neuroscience Research, vol. 90, no. 11, pp. 21932200, 2012.

[42] S. Delatorre, G. Rojas-Piloni, G. Martinez-Lorenzana, J. Rodriguez-Jimenez, L. Villanueva, and M. Condes-Lara, "Paraventricular oxytocinergic hypothalamic prevention or interruption of long-term potentiation in dorsal horn nociceptive neurons: electrophysiological and behavioral evidence," Pain, vol. 144, no. 3, pp. 320-328, 2009.

[43] M. Condes-Lara, G. Rojas-Piloni, G. Martinez-Lorenzana, J. Rodriguez-Jimenez, M. Lopez Hidalgo, and M. J. FreundMercier, "Paraventricular hypothalamic influences on spinal nociceptive processing," Brain Research, vol. 1081, no. 1, pp. 126-137, 2006.

[44] J. Hu, Z. Wang, Y. Y. Guo et al., "A role of periaqueductal grey NR2B-containing NMDA receptor in mediating persistent inflammatory pain," Molecular Pain, vol. 5, pp. 71-81, 2009.

[45] J. A. Harris, "Using c-fos as a neural marker of pain," Brain Research Bulletin, vol. 45, no. 1, pp. 1-8, 1998.

[46] M. Lehner, E. Taracha, A. Skorzewska et al., "Sensitivity to pain and c-Fos expression in brain structures in rats," Neuroscience Letters, vol. 370, no. 1, pp. 74-79, 2004. 

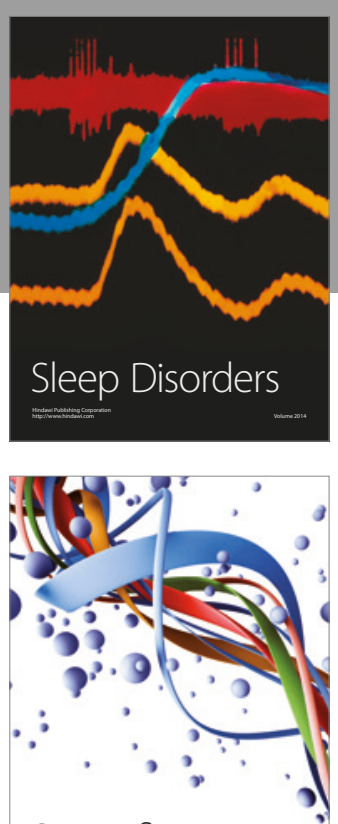

Scientifica
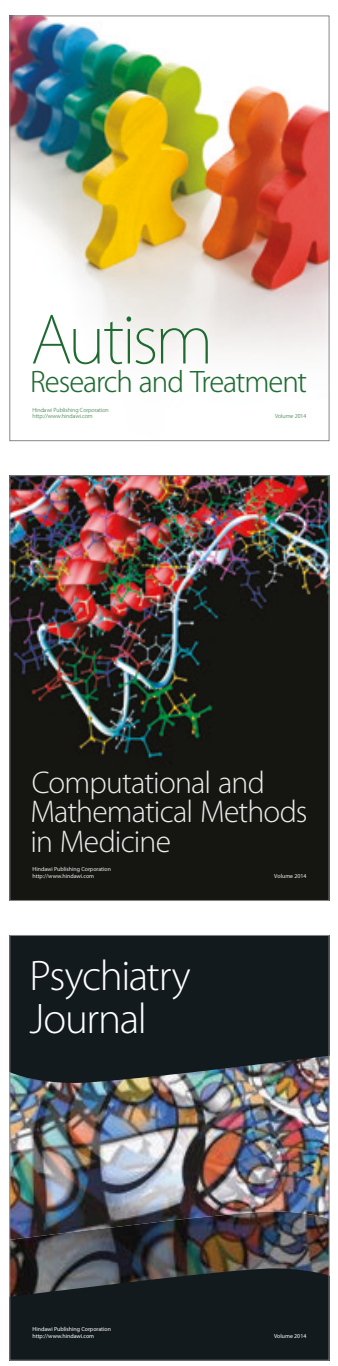
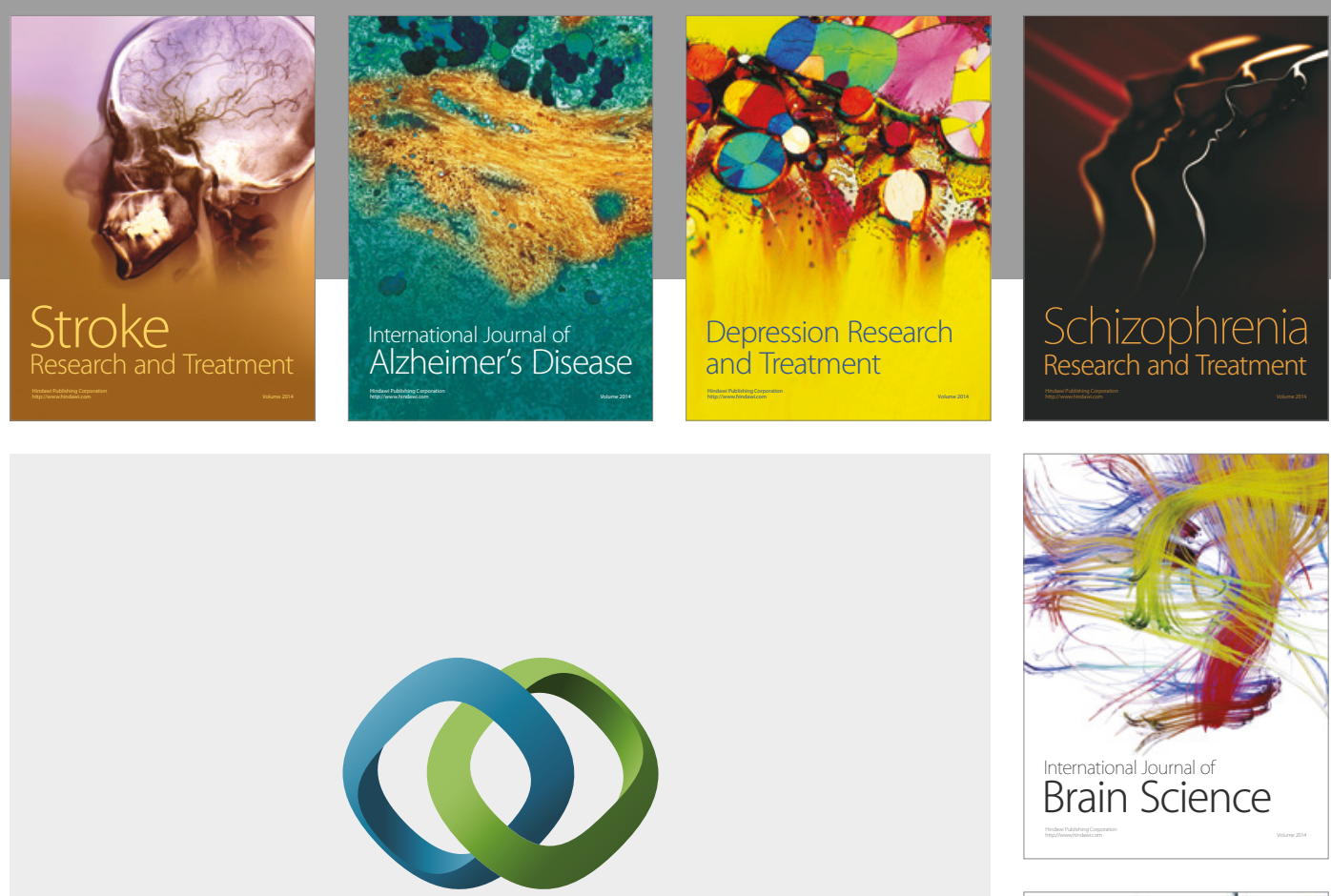

\section{Hindawi}

Submit your manuscripts at

https://www.hindawi.com
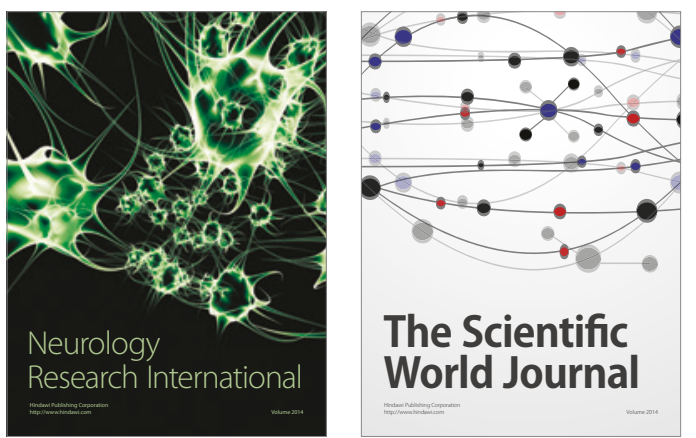

The Scientific World Journal

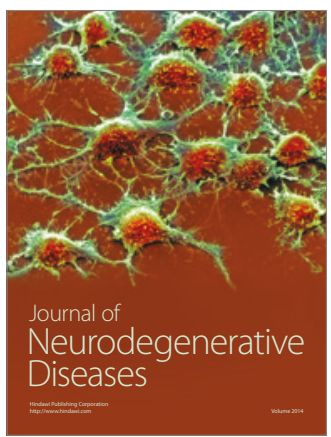

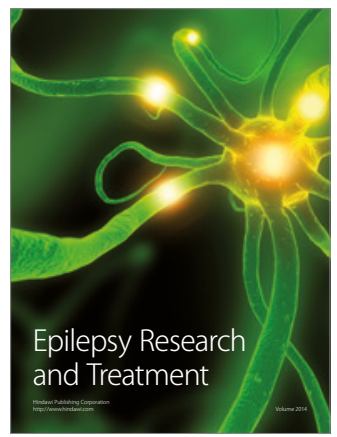

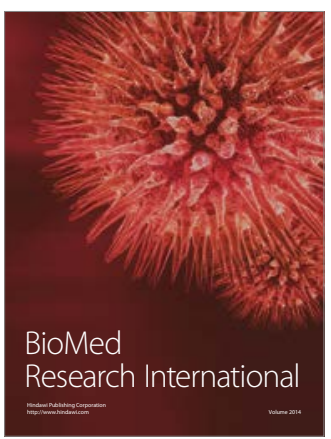

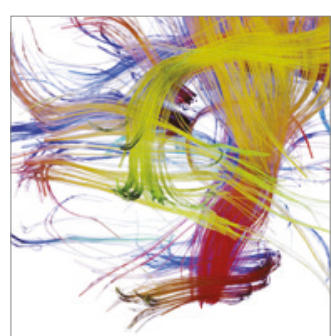

Brain Science

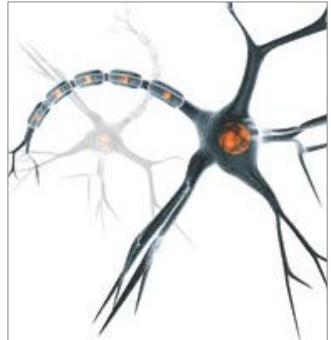

Neural Plasticity
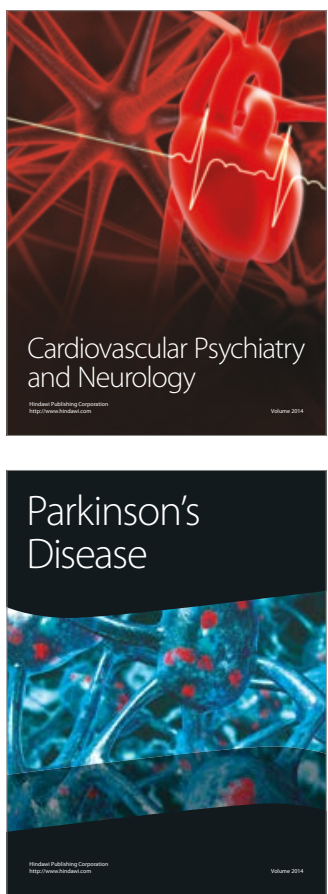